\title{
BMJ Open Feasibility and effect of home-based therapy programmes for children with cerebral palsy: a protocol for a systematic review
}

\author{
L W M E Beckers, ${ }^{1,2}$ M L A P Schnackers, ${ }^{3,4}$ Y J Janssen-Potten, ${ }^{1,2} \mathrm{~J}$ Kleijnen, ${ }^{5,6}$ \\ B Steenbergen ${ }^{3,7}$
}

To cite: Beckers LWME, Schnackers MLAP, JanssenPotten YJ, et al. Feasibility and effect of home-based therapy programmes for children with cerebral palsy: a protocol for a systematic review. BMJ Open 2017;7: e013687. doi:10.1136/ bmjopen-2016-013687

- Prepublication history for this paper is available online. To view these files please visit the journal online (http://dx.doi.org/10.1136/ bmjopen-2016-013687).

MLAPS contributed equally.

Received 29 July 2016 Revised 6 December 2016 Accepted 22 December 2016

CrossMark

For numbered affiliations see end of article.

Correspondence to

L W M E Beckers;

laura.beckers@

maastrichtuniversity.nl

\section{ABSTRACT \\ Introduction: Given the promising advantages of upper extremity home-based programmes in children with cerebral palsy (CP), a systematic review of the available literature on this topic is warranted. The purpose of the systematic review described in this protocol is to investigate currently available home-based occupational therapy and physiotherapy programmes regarding both their feasibility and effect.}

Methods and analysis: This protocol describes a systematic review, developed in accordance with the Preferred Reporting Items for Systematic Review and Meta-Analysis Protocols (PRISMA-P) 2015. Studies will be included in which primary data are collected, participants are children aged $<18$ years with any type of $\mathrm{CP}$ and the intervention of interest is a home-based occupational therapy or physiotherapy intervention. Comparators of interest are: no therapy, care as usual, centre-based occupational therapy or physiotherapy, an alternative home-based programme and a medical intervention. Studies will be included that report either on feasibility (ie, acceptability, demand,

implementation, practicality, adaptation, expansion or integration) or on efficacy/effectiveness (ie, childrelated upper extremity outcomes within all International Classification of Functioning, Disability and Health levels or parent-related/caregiver-related outcomes on the psychological and social domain). Relevant studies will be identified by searching the databases MEDLINE, EMBASE, CINAHL, PsycINFO, PEDro, OTSeeker and CPCI-S as well as the trial registers ICTRP and CENTRAL, the reference lists of included records and by circulating a bibliography of the included records to authors of included studies. There will be no restrictions on language or year of publication. The search strategy consists of terms related to the population and intervention. Data will be extracted in duplicate using a digital data extraction form.

Ethics and dissemination: The proposed study does not involve collection of primary data. Accordingly, no ethical approval is required. The authors will disseminate the findings of this systematic

\section{Strengths and limitations of this study}

- The systematic review described in this protocol will be the first review to be systematic as well as specifically focused on home-based occupational therapy and physiotherapy programmes in children with cerebral palsy.

- By systematic review, feasibility as well as effect will be investigated.

- In the systematic review, both child-related and parent-related outcomes will be included.

- During the systematic review, selection of records, data collection, assessment of the risk of bias and judgement of the quality of evidence will be performed in duplicate, independently by two reviewers.

- It is anticipated that no meta-analysis can be conducted due to the expected clinical and methodological heterogeneity of reports.

review through publication in a peer-reviewed journal and conference presentation(s).

Trial registration number: CRD42016043743; pre-results.

\section{INTRODUCTION}

Cerebral palsy (CP) is one of the most common causes of physical disability in children. The majority of children with CP have impaired arm-hand function. ${ }^{1}$ Abundant research has shown the effectiveness of centre-based therapies in children with CP, including upper extremity interventions such as constraint-induced movement therapy and bimanual training. ${ }^{2-5}$

In recent years, home-based programmes have received increasing attention in rehabilitation of children with CP. These programmes 
are a useful addition to centre-based occupational therapy and physiotherapy for a number of reasons. First, home-based programmes provide a unique opportunity to continue aspects of therapy, either in between centre-based sessions or after centre-based therapy has ended. This benefits the retention of established intervention effects. Second, they increase parental involvement and empowerment, in turn contributing to reciprocal partnerships between parents and health professionals. This enables parents and health professionals to learn from each other and share each other's perspectives on the rehabilitation of the child. Third, homebased programmes anticipate changes in the healthcare system by providing a treatment approach that is considered to be cost-effective. Fourth, and finally, home-based programmes may be the preferred or even the only feasible option in specific contexts, for example, in cases where long distances need to be travelled from the child's home to the institution. Given these promising advantages of home-based programmes in children with $\mathrm{CP}$, a systematic review of the available literature on this topic is warranted.

In a recent study, Sakzewski et at provided a systematic overview of non-surgical upper extremity therapies in children with unilateral CP and argued that treatment at home may be an effective supplement to centre-based interventions. In the same year, Novak and Berry ${ }^{7}$ focused on the effectiveness of home-based programmes in children with CP. In contrast to the study of Sakzewski et al, the review of Novak $e t$ al was not systematic. Similar to Sakzewski et al however, they concluded that homebased programmes using goal directed training are effective for improving motor outcomes. ${ }^{7}$ To extend the two previous studies, the review described in this protocol will be systematic as well as specifically focused on home-based interventions in children with CP. The review will be distinctive because its purpose is to investigate currently available home-based occupational therapy and physiotherapy programmes regarding both their feasibility and their effect, and to evaluate both child-related and parent-related outcomes.

Feasibility will be addressed, as this is a prerequisite for effective home-based programmes. Since feasibility is a general facet of home-based programmes per se, the review will not be limited to feasibility of upper extremity programmes. Feasibility of a variety of home-based occupational therapy and physiotherapy programmes will be reviewed. In contrast, the review of the effect will indeed be limited to home-based programmes that focus on the upper extremity.

Both efficacy and effectiveness will be addressed. Efficacy and effectiveness are related concepts, but with an important difference between them. Efficacy relates to results of an intervention under ideal circumstances (ie, explanatory study). Effectiveness, on the other hand, relates to the beneficial effect of an intervention under 'real world' clinical settings (ie, pragmatic study). ${ }^{8}$ Parents play a key role in a home-based programme for their child. Hence, their contribution to the programme might affect their psychosocial health, either positive or negative, which may influence the adherence to the programme. Therefore, next to child-related outcomes, parent-related outcomes are of primary interest in our evaluation of efficacy and effectiveness of home-based programmes.

Comparison of effectiveness of different upper extremity home-based programmes is critical for clinical decision-making when considering options for individual treatment plans, or for institutional decision-making. Moreover, detailed insight into different home-based programmes regarding feasibility, efficacy as well as effectiveness will result in recommendations to improve existing home-based programmes and to develop and design of new programmes.

\section{Objectives}

The aim of the systematic review described in this protocol is to provide a clear view on the available homebased occupational therapy and physiotherapy programmes in children with CP (aged $<18$ years), specifically home-based programmes that focus on the upper extremity. Two objectives will be addressed by the systematic review:

1. To assess the feasibility of home-based occupational therapy and physiotherapy programmes in children with CP.

2. To assess the efficacy and effectiveness of home-based occupational therapy and physiotherapy programmes that focus on the upper extremity in children with $\mathrm{CP}$, on child-related and parent-related outcomes.

\section{METHODS AND ANALYSIS \\ Design}

The protocol for the systematic review was developed in accordance with the Preferred Reporting Items for Systematic Review and Meta-Analysis Protocols (PRISMA-P) 2015. ${ }^{9} 10$

\section{Eligibility criteria}

\section{Study designs}

Studies in which primary data were collected will be included. That is, (systematic) reviews and meta-analyses will be excluded. A relatively small number of available studies are expected. ${ }^{7}$ Since it is strived for an overview of all available evidence, all types of study designs will be included regarding feasibility as well as efficacy and effectiveness. However, the hierarchy of evidence will be taken into consideration for reporting of the results of the review.

\section{Participants}

Studies on children aged $<18$ years with any type of $\mathrm{CP}$ will be included. Studies in which adult patients participated in the home-based programme next to children will also be included, provided that data were reported 
separately for children and adults (aged $\geq 18$ years). Likewise, studies in which both children with CP and children with other disorders were studied will also be included, provided that data of children with CP were reported separately.

\section{Interventions and setting}

Studies that report on at least one home-based occupational therapy or physiotherapy intervention will be included. An intervention is considered to be homebased if treatment is performed in the home setting without a healthcare provider being physically present. Studies that only include therapy provided at a healthcare facility, (pre)school or day care will be excluded. In case the intervention takes place in different settings, studies will be included if treatment of the child in the home setting is a fundamental, prespecified element of the intervention. Regarding the research objective on efficacy/effectiveness, exclusively studies that report on interventions targeting the upper extremity will be included. Hence, studies that report on general gross motor function will be excluded.

We consider home-based programmes as complex interventions, composed of several interacting components. Possible components could be, for example, training of the parents, exercises or supervision by a healthcare professional. Where possible, feasibility, efficacy or effectiveness of components will be described separately.

\section{Comparators}

The nature of the comparator (ie, the intervention against which the home-based programme is compared) is unrelated to the feasibility of the home-based programme. Hence, to describe the feasibility of home-based programmes, studies on all possible control interventions will be included as well as studies without one.

To assess efficacy/effectiveness, comparators of interest are: no therapy, care as usual, centre-based occupational therapy or physiotherapy, and medical intervention (ie, drugs or a surgical procedure such as botulinum toxin injections). Studies in which two or more home-based programmes were compared with each other will also be included.

\section{Outcomes}

To determine feasibility, studies will be included that reported on outcomes within the areas acceptability, demand, implementation, practicality, adaptation, expansion and integration. ${ }^{11}$ These terms will be elaborated on in the paragraph on 'Outcomes and prioritisation'.

With regard to efficacy and effectiveness, the outcomes of interest are:

- Child-related outcomes within all levels of the International Classification of Functioning, Disability and Health (ICF), that is, body functions and structures, activity and participation. ${ }^{12}$
- Parent-related or caregiver-related outcomes on the psychological and social domain.

Studies reporting on at least one of the aforementioned outcomes regarding feasibility, efficacy or effectiveness will be included.

\section{Timing}

There will be no restrictions on the length of follow-up to assess outcomes.

\section{Language}

There will be no language restrictions.

\section{Information sources}

Several complementary information sources will be used to identify relevant studies, applying the strategy described in the next paragraph. Also, unpublished studies will be searched for. There will be no restriction on the year of publication. First, the following electronic databases will be searched for literature: MEDLINE (Ovid interface), EMBASE (Ovid interface) CINAHL (EBSCO interface), PsycINFO (EBSCO interface), CPCI-S (Web of Science interface), OTseeker and PEDro. The search will be supplemented by searching for trial protocols through the trial register ICTRP. Until reaching a point of literature saturation (ie, the moment when no new relevant studies emerge), the reference lists of included records will be scrutinised, as well as the reference lists of (systematic) reviews and meta-analysis that were found during the search. Titles and abstracts of the references will be compared with the eligibility criteria to decide on inclusion in the selection process. Finally, a bibliography of the included records will be circulated to all corresponding and last authors of included studies. They will be asked to provide details of any other related study either by their research group or associates.

\section{Search strategy}

The search strategy includes keywords (eg, MESH-terms) and text words (ie, within title and abstract), combining population and intervention-related search terms, for example:

\section{MEDLINE search—Ovid interface}

1. Cerebral Palsy/

2. ((cerebral adj2 pals\$) or encephalopathia infantalis or spastic diplegia $\$$ or little $\$$ disease).ti,ab.

3. or $/ 1-2$

4. exp Self Care/

5. Home Care Services/

6. (Home or in?home or home?based or self care or residence or domiciliary).ti,ab.

7. or $/ 4-6$

8. exp Exercise Therapy/

9. Physical Therapy Modalities/

10. (Exercise $\$$ or therapy or therapies or program $\$$ or train $\$$ or physiotherapy $\$$ or occupational or (physical adj2 therap\$)).ti,ab. 
11. or $/ 8-10$

12. 3 and 7 and 11

Two members of the research team (LWMEB and JK) developed the search strategy for each database and trial register. JK will perform the search.

\section{Study records}

Data management

Literature search results and corresponding PDF files will be uploaded to the reference management software EndNote. EndNote will be used to remove duplicate records. All records will be uploaded in Covidence. This program will be used for selection, data collection and maintenance of the review.

\section{Selection process}

Before the selection process starts, search results of the various information sources will be merged and duplicate records will be removed. The first phase of the selection process consists of the screening of titles and abstracts on eligibility criteria such that irrelevant records are removed. In case of any doubt regarding relevance, the record will remain included at this stage. Subsequently, full texts will be retrieved for all potentially relevant records. Full-text records will be examined for compliance of studies with the eligibility criteria. Records for which the full text cannot be retrieved may also be included, although results will be reported separately. Multiple records of the same study will be identified and handled as described in the paragraph 'data collection process'.

The first phase of the selection process (ie, screening of titles and abstracts) will be performed in duplicate by two independent reviewers (LWMEB and MLAPS). Examination of the full-text records will be performed in duplicate and independently by the same reviewers (LWMEB and MLAPS), who will not be blinded to any study information. Inter-rater agreement will be calculated. In case of discrepancies in any phase of the selection process, a third and fourth reviewer (YJJ-P and BS) will mediate to reach consensus.

\section{Data collection process}

Data extraction will be carried out by use of Covidence, in duplicate by two independent reviewers (LWMEB and MLAPS). To increase consistency between the reviewers, the data extraction form will be pilot tested before the start of the data collection process of the review. This will be carried out by duplicate completion of the form for two CP-related records (one feasibility study and one randomised controlled trial), which are not eligible for inclusion in this review (LWMEB and MLAPS). Adaptations will be processed based on discrepancy between the data extraction results of the reviewers, evaluated by a third reviewer (YJJ-P) and the reviewers' experiences during the pilot test. In case of discrepancies between the reviewers during the review, a third and fourth reviewer (YJJ-P and $\mathrm{BS}$ ) will arbitrate to reach consensus.

In the absence of complete descriptions of essential information, the reviewers will contact the corresponding author to collect the required information. The decision to contact an author will be made in mutual agreement between the four reviewers (LWMEB, MLAPS, YJJ-P and BS). On the basis of an earlier study, it is expected to receive additional information for $\sim 50 \%$ of the incompletely described studies. ${ }^{13}$

Multiple records of a single study will be searched for by comparing author names, intervention locations, intervention characteristics, sample sizes and outcomes of eligible records. If multiple records present different outcome variables or time points, they will be combined into one record within the review. If overlapping records on the same outcome variable(s) as well as the same time points are found, only the one reporting on the largest sample size will be included in the review. This will avoid double-counting of participants. In case of inconsistencies between reports, the reviewers will contact the authors for clarification, using the same approach mentioned earlier in this paragraph.

\section{Data items}

From all records, the following information will be extracted: author(s), publication date, study design, country, comparator, number of participants (in total and per study arm), outcomes, duration of follow-up and time points of measurements.

The following treatment characteristics of the homebased programmes will be extracted: objective, therapy provider(s), duration of the programme, frequency and duration of sessions, treatment approach (eg, taskspecific training). Furthermore, demographics of participating children will be extracted: age, gender, diagnosis (including type and topographical distribution of CP), level on the Manual Ability Classification System, level on the Gross Motor Function Classification System and level on the Communication Function Classification System. Finally, the demographics of parents of participating children will be extracted: age, gender and educational level. The data extracted from the included studies will be summarised and tabulated.

\section{Outcomes and prioritisation}

Concerning the feasibility objective, the following areas of outcomes will be applied: ${ }^{11}$

Primary outcome:

- Acceptability: 'the extent to which programme deliverers or programme recipients judge the programme as suitable, satisfying or attractive (eg, satisfaction)'. Secondary outcomes:

- Demand: 'the extent to which a programme is likely to be used (eg, expressed interest to use)'.

- Implementation: "the extent to which a programme can successfully be delivered to intended participants in 
some defined, but not fully controlled, context (eg, success or failure of execution)'.

- Practicality: 'the extent to which a programme can be carried out with intended participants using existing means, resources and circumstances and without outside intervention (eg, ability of participants to carry out intervention activities)'.

- Adaptation: 'the extent to which an existing programme performs when changes are made regarding format or population (eg, degree to which similar outcomes are obtained in a new format)'.

- Integration: 'the extent to which a programme can be integrated within an existing system (eg, perceived sustainability)'.

- Expansion: "the extent to which a previously tested programme can be expanded to provide a new programme or service (eg, positive or negative effects on organisation)'.

For the efficacy and effectiveness studies, the following outcomes will be used:

Primary outcomes:

- Child-related upper extremity outcomes within the level activity of the ICF (eg, Assisting Hand Assessment). Secondary outcomes:

- Child-related upper extremity outcomes within the levels body functions and structures and participation of the ICF (eg, spasticity assessment and Children's Assessment of Participation and Enjoyment, respectively).

- Parent-related or caregiver-related outcomes within the psychological and social domain (eg, Parenting Stress Index).

If available, results from an intention-to-treat analysis will be used. All outcomes may be measured quantitatively or qualitatively.

\section{Risk of bias individual studies}

Risk of bias of predominantly quantitative studies will be assessed by the Checklist for Measuring Quality by Downs and Black. ${ }^{14}$ The checklist contains 27 yes/no questions across five constructs: study quality (the overall quality of the study); external validity (the ability to generalise findings of the study); study bias (to assess bias in the intervention and outcome measure(s)); confounding and selection bias (to determine bias from sampling or group assignment); and power of the study (to determine if findings are due to chance). ${ }^{14}$ Risk of bias of predominantly qualitative studies will be assessed by the JBI Critical Appraisal Checklist for Qualitative Research, which consists of 10 questions that can be answered with yes, no, unclear or not applicable. ${ }^{15}$

The risk of bias will be assessed for all applicable studies in duplicate by two independent reviewers (LWMEB and MLAPS), without blinding. To increase consistency between the reviewers, the checklists will be pilot tested before the start of the risk of bias assessment of the review. This will be carried out by duplicate assessment of two CP-related records (one predominantly quantitative study and one predominantly qualitative study), which are not eligible for inclusion in this review. In case of disagreement between reviewers during the review, a third and fourth reviewer (YJJ-P and BS) will arbitrate. Risk of bias will be assessed on the study level. Results across studies will be presented graphically. Additionally, the reviewers will state how the risk of bias may have influenced their review findings.

\section{Data synthesis}

Based on the results of the article of Novak et $a l^{7}$ a limited number of efficacy and effectiveness studies are expected to be found by the systematic search. Additionally, the studies are expected to be both clinically and methodologically heterogeneous. Hence, it is anticipated that conducting a meta-analysis will not be appropriate. Therefore, a method for meta-analysis is not included in this protocol. If the assumption emerges to be wrong, this will result in an amendment of the protocol, in which a method for meta-analysis will be included. An amendment will be made if at least three efficacy and effectiveness studies are comparable regarding treatment, comparator(s) and outcome $(\mathrm{s}) .{ }^{16}$ The reviewers (LWMEB, MLAPS, YJJ-P and BS) will decide whether a meta-analysis is appropriate and report the rationale.

\section{Meta-biases}

For the efficacy and effectiveness studies included in the review, the risk of selective reporting (outcome reporting bias) will be determined. This will be assessed by comparing the records on study results with previously published study protocols and registrations. The studies of which no study protocol or trial registration was found will also be listed. This procedure will be performed by one reviewer (LWMEB).

In case of $\geq 10$ homogeneous efficacy and effectiveness studies (according to the criteria described in the paragraph 'data synthesis'), risk of publication bias will be assessed by graphing a funnel plot and, if appropriate, supplemented by a statistical test. In the plot, it will be indicated which studies have been published in a journal and which ones have not been (yet).

\section{Confidence in cumulative estimate}

For each efficacy and effectiveness outcome, the Grading of Recommendations Assessment, Development and Evaluation (GRADE) guidelines will be used to judge the quality of evidence within the domains risk of bias, publication bias, imprecision, inconsistency and indirectness. ${ }^{17}$ The quality of the evidence can be scored as high quality (very confident that the true effect lies close to that of the estimate of the effect), moderate quality (moderately confident in the effect estimate: the true effect is likely to be close to the estimate of the effect, but there is a possibility that it is substantially different), low quality (confidence in the effect estimate is limited: the true effect may be substantially 
different from the estimate of the effect) or very low quality (very little confidence in the effect estimate: the true effect is likely to be substantially different from the estimate of effect). The same approach as described in the paragraph 'risk of bias individual studies' will be used for pilot testing and scoring.

\section{Amendments}

In case of protocol amendments, the authors will document the date, description of the changes and rationale for each amendment.

\section{ETHICS AND DISSEMINATION Ethical considerations}

The proposed study does not involve collection of primary data. Accordingly, no ethical approval is required.

\section{DISSEMINATION PLAN}

This systematic review protocol was registered in the International Prospective Register of Systematic Reviews (PROSPERO) on 29 July 2016 (registration number CRD42016043743).

The authors will disseminate the findings of this review through publication in a peer-reviewed journal and conference presentation(s). The results will be reported according to the most recent version of the Preferred Reporting Items for Systematic Reviews and Meta-Analyses (PRISMA). ${ }^{18}$

\section{Author affiliations}

${ }^{1}$ Department of Rehabilitation Medicine, School for Public Health and Primary Care (CAPHRI), Maastricht University, Maastricht, The Netherlands

${ }^{2}$ Adelante, Center of Expertise in Rehabilitation and Audiology, Hoensbroek, The Netherlands

${ }^{3}$ Radboud University Nijmegen, Behavioural Science Institute, Nijmegen, The Netherlands

${ }^{4}$ Department of Rehabilitation, Radboud University Medical Centre, Nijmegen, The Netherlands

${ }^{5}$ School for Public Health and Primary Care (CAPHRI), Maastricht University, Maastricht, The Netherlands

${ }^{6}$ Kleijnen Systematic Reviews Ltd, York, UK

${ }^{7}$ Australian Catholic University, School of Psychology, Melbourne, Australia

Contributors LWMEB designed the study protocol and drafted the initial manuscript. LWMEB and JK developed the search strategy. MLAPS, YJJ-P, JK and BS provided critical insights and reviewed the protocol and manuscript, making important intellectual contributions. All authors read and approved the final version.

Funding This work was supported by ZonMw (the Netherlands Organisation for Health Research and Development), grant number 630000001, as part of the research project COAD (Co-creation at hand: The road to independence). The clinical trial that is part of the COAD-project is registered under number NTR5743.

Competing interests None declared.

Provenance and peer review Not commissioned; externally peer reviewed.

Open Access This is an Open Access article distributed in accordance with the Creative Commons Attribution Non Commercial (CC BY-NC 4.0) license, which permits others to distribute, remix, adapt, build upon this work noncommercially, and license their derivative works on different terms, provided the original work is properly cited and the use is non-commercial. See: http:// creativecommons.org/licenses/by-nc/4.0/

\section{REFERENCES}

1. Arner M, Eliasson AC, Nicklasson S, et al. Hand function in cerebral palsy. Report of children in a population-based longitudinal healthcare program. J Hand Surg 2008;33: 1337-47.

2. Novak I, Mclntyre S, Morgan C, et al. A systematic review of interventions for children with cerebral palsy: state of the evidence. Dev Med Child Neurol 2013;55:885-910.

3. Aarts PB, Jongerius PH, Geerdink YA, et al. Effectiveness of modified constraint-induced movement therapy in children with unilateral spastic cerebral palsy: a randomized controlled trial. Neurorehabil Neural Repair 2010;24:509-18.

4. Gordon AM, Schneider JA, Chinnan A, et al. Efficacy of a hand-arm bimanual intensive therapy (HABIT) in children with hemiplegic cerebral palsy: a randomized control trial. Dev Med Child Neurol 2007;49:830-8.

5. de Brito Brandao M, Gordon AM, Mancini MC. Functional impact of constraint therapy and bimanual training in children with cerebral palsy: a randomized controlled trial. Am J Occup Ther 2012;66:672-81.

6. Sakzewski L, Ziviani J, Boyd RN. Efficacy of upper limb therapies for unilateral cerebral palsy: a meta-analysis. Pediatrics 2014;133: e175-204.

7. Novak I, Berry J. Home program intervention effectiveness evidence. Phys Occup Ther Pediatr 2014;34:384-9.

8. Gartlehner G, Hansen RA, Nissman D, et al. Criteria for distinguishing effectiveness from efficacy trials in systematic reviews. Technical Review 12 (Prepared by the RTI-International-University of North Carolina Evidence-based Practice Center under Contract No. 290-02-0016.) AHRQ Publication No. 06-0046. Rockville, MD: Agency for Healthcare Research and Quality, 2006.

9. Moher D, Shamseer L, Clarke M, et al. Preferred reporting items for systematic review and meta-analysis protocols (PRISMA-P) 2015 statement. Syst Rev 2015;4:1.

10. Shamseer L, Moher D, Clarke M, et al. Preferred reporting items for systematic review and meta-analysis protocols (PRISMA-P) 2015: elaboration and explanation. BMJ 2015;349:97647.

11. Bowen DJ, Kreuter M, Spring B, et al. How we design feasibility studies. Am J Prev Med 2009;36:452-7.

12. World Health Organization. Towards a common language for functioning, disability and health. ICF: the international classification of functioning, disability and health. Geneva: World Health Organization, 2002. http://www.who.int/classifications/icf/icfbeginnersguide.pdf (accessed Jul 2016).

13. Selph SS, Ginsburg AD, Chou R. Impact of contacting study authors to obtain additional data for systematic reviews: diagnostic accuracy studies for hepatic fibrosis. Syst Rev 2014:3:107.

14. Downs SH, Black N. The feasibility of creating a checklist for the assessment of the methodological quality both of randomised and non-randomised studies of healthcare interventions. J Epidemiol Community Health 1998;52:377-84.

15. The Joanna Briggs Institute. The Joanna Briggs Institute Critical Appraisal tools for use in JBI systematic reviews. Checklist for qualitative research. The Joanna Briggs Institute, 2016. http:// joannabriggs.org/assets/docs/critical-appraisal-tools/JBI_Critical Appraisal-Checklist_for_Qualitative_Research.pdf (accessed Jul 2016).

16. Higgins JPT, Green S, eds. Cochrane handbook for systematic reviews of interventions version 5.1.0 [updated March 2011]. The Cochrane Collaboration, 2011. http://handbook.cochrane.org/ (accessed Jul 2016).

17. Guyatt GH, Oxman AD, Schunemann HJ, et al. GRADE guidelines: a new series of articles in the journal of clinical epidemiology. $J$ Clin Epidemiol 2011;64:380-2.

18. Moher D, Liberati A, Tetzlaff J, et al. Preferred reporting items for systematic reviews and meta-analyses: the PRISMA statement. Int J Surg 2010;8:336-41. 